Published in The Astrophysical Journal, Volume 756, Issue 1, ARTiCle ID. 49 (2012)

Preprint typeset using $\mathrm{LATE}_{\mathrm{E}} \mathrm{X}$ style emulateapj v. 5/2/11

\title{
HIGH-RESOLUTION X-RAY SPECTROSCOPY OF THE GALACTIC SUPERNOVA REMNANT PUPPIS A WITH THE XMM-NEWTON RGS
}

\author{
Satoru Katsuda ${ }^{1}$, Hiroshi Tsunemi ${ }^{2}$, Koji Mori ${ }^{3}$, Hiroyuki Uchida ${ }^{4}$, Robert Petre ${ }^{5}$, \\ Shin'ya Yamada ${ }^{1}$, Hiroki Akamatsu ${ }^{6}$, SaOri Konami ${ }^{1,7}$, and Toru Tamagawa ${ }^{1}$ \\ Published in The Astrophysical Journal, Volume 756, Issue 1, article id. 49 (2012)
}

\begin{abstract}
We present high-resolution X-ray spectra of cloud-shock interaction regions in the eastern and northern rims of the Galactic supernova remnant Puppis A, using the Reflection Grating Spectrometer onboard the XMM-Newton satellite. A number of emission lines including $\mathrm{K} \alpha$ triplets of He-like N, $\mathrm{O}$, and $\mathrm{Ne}$ are clearly resolved for the first time. Intensity ratios of forbidden to resonance lines in the triplets are found to be higher than predictions by thermal emission models having plausible plasma parameters. The anomalous line ratios cannot be reproduced by effects of resonance scattering, recombination, or inner-shell ionization processes, but could be explained by charge-exchange emission that should arise at interfaces between the cold/warm clouds and the hot plasma. Our observations thus provide observational support for charge-exchange X-ray emission in supernova remnants.

Subject headings: atomic processes - ISM: abundances — ISM: individual objects: Puppis A — ISM: supernova remnants - X-rays: ISM
\end{abstract}

\section{INTRODUCTION}

It has long been thought that the X-ray emission from supernova remnants (SNRs) is partly due to charge-exchange (CX) processes between neutrals and highly-ionized ions (e.g., Serlemitsos et al. 1973). Wise \& Sarazin (1989) first performed a detailed theoretical calculation of CX-induced X-ray emission from SNRs. They found that the contribution of CX emission is typically $10^{-3}$ to $10^{-5}$ compared with that of thermal emission (i.e., electron collisional excitation lines). This means that CX emission is in general very minor. However, as the authors claimed, CX emission could play an enhanced role where neutrals are mixed with shocked hot gas because of hydrodynamic instabilities in SNRs and/or inhomogeneities of the interstellar medium. Subsequently, Lallement (2004) examined projected emission profiles for both CX and thermal emission in SNRs, and noted that CX X-ray emission could be comparable with thermal emission in thin layers at the SNR edge. It is also pointed out that the relative importance of CX X-ray emission to thermal emission is proportional to a quantity, $n_{\mathrm{c}} V_{\mathrm{r}} n_{\mathrm{e}}^{-2}$, with $n_{\mathrm{c}}$ being the cloud density, $V_{\mathrm{r}}$ the relative velocity between neutrals and ions, and $n_{\mathrm{e}}$ the electron density of the hot plasma. Thus, the higher the density contrast $\left(n_{\mathrm{c}} / n_{\mathrm{e}}\right)$, the stronger the presence of $\mathrm{CX}$

\footnotetext{
${ }^{1}$ RIKEN (The Institute of Physical and Chemical Research), 2-1 Hirosawa, Wako, Saitama 351-0198

${ }^{2}$ Department of Earth and Space Science, Graduate School of Science, Osaka University, 1-1 Machikaneyama, Toyonaka, Osaka, 60-0043, Japan

${ }^{3}$ Department of Applied Physics and Electronic Engineering, Faculty of Engineering, University of Miyazaki, 1-1 Gakuen Kibanadai-Nishi, Miyazaki, 889-2192, Japan

${ }^{4}$ Department of Physics, Kyoto University, Kitashirakawaoiwake-cho, Sakyo, Kyoto 606-8502, Japan

5 NASA Goddard Space Flight Center, Code 662, Greenbelt MD 20771

${ }^{6}$ Department of Physics, Tokyo Metropolitan University, 1-1 Minami-Osawa, Hachioji, Tokyo 192-0397

${ }^{7}$ Department of Physics, Tokyo University of Science, 1-3 Kagurazaka, Shinjuku-ku, Tokyo 162-8601
}

X-ray emission would become.

Observational evidence of CX emission from SNRs was found at optical wavelengths more than $30 \mathrm{yrs}$ ago (e.g., Kirshner \& Chevalier 1978), but is still lacking in the $\mathrm{X}$-ray domain. So far, only marginal detections of CX X-ray emission were reported in the SMC SNR 1E0102.27219 (Rasmussen et al. 2001), and the Galactic SNR, the Cygnus Loop (Katsuda et al. 2011). The line intensity ratios of $(n>2 \rightarrow n=1) /(n=2 \rightarrow n=1)$ in H-like O (1E0102.2-7219) or He-like O (Cygnus Loop) seem to be higher than expectations from thermal emission models, and were interpreted as signatures of CX. However, the CX interpretation is still a matter of debate, because Chandra grating observations of 1E0102.27219 (Flanagan et al. 2004) did not confirm the XMMNewton result, and uncertainties of Fe L line emissivities might cause the spectral anomaly in the Cygnus Loop (Katsuda et al. 2011).

One SNR that might show CX X-ray emission is Puppis A, a large $\left(\sim 50^{\prime}\right.$ in diameter $)$ middle-aged $(\sim 3700 \mathrm{yr}$ : Winkler et al. 1988) SNR in our Galaxy. Its surface brightness is the highest among all Galactic SNRs in soft X-rays $(E<1 \mathrm{keV})$. The X-ray image is inhomogeneous with particularly bright eastern and northern knots (hereafter, BEK and NK). These regions undoubtedly represent strong interactions between the SNR blast wave and dense clouds (e.g., Petre et al. 1982; Dubner \& Arnal 1988; Hwang et al. 2005), and thus they exhibit ideal conditions in which CX X-ray emission could be significant (Lallement 2004). While indications of CX signatures have not yet been reported in this SNR, such signatures can be best obtained through high-resolution X-ray spectroscopy.

Here, we present high-resolution X-ray spectra of the BEK/NK features using XMM-Newton's Reflection Grating Spectrometer (RGS: den Herder et al. 2001). From the RGS spectra, forbidden-to-resonance line ratios in He $\alpha$ triplets are found to be anomalously enhanced, especially at the BEK. We show that this anomaly could 

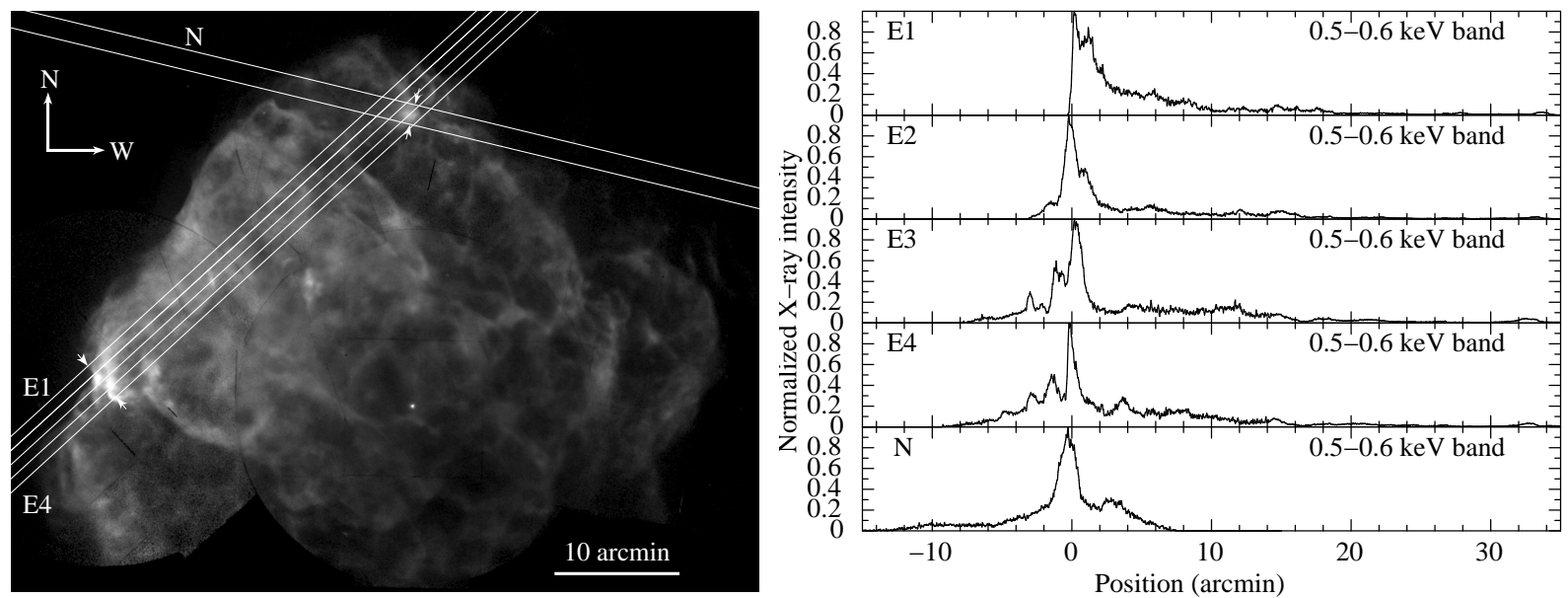

FIG. 1.- Left: Merged XMM-Newton and Chandra image in the energy band $0.5-5 \mathrm{keV}$. The intensity scales as the square root of the surface brightness. The RGS spectral extraction regions are overlaid in white with the region name. The arrows indicate zero points of projection profiles shown in Fig. 1 right. Right: X-ray emission profiles along the RGS dispersion axis for the five spectral extraction regions. The zero points $(\mathrm{x}=0)$ correspond to the direction from which emission is detected at the nominal (no red/blue shift) wavelength positions on the RGS detectors.

be naturally caused by the presence of CX emission.

\section{OBSERVATIONS AND SPECTRAL ANALYSIS}

The NK and BEK were observed by XMM-Newton on 2003 April 17 (Obs.ID 0150150101) and 2003 May 21 (Obs.IDs 0150150201 and 0150150301), respectively, in order to obtain high-resolution spectra using the RGS. The dispersion directions of the RGS are $76^{\circ} .4(\mathrm{NK})$ and $132^{\circ} .3$ (BEK) counterclockwise from the north, as shown in Fig. 1, which is an X-ray image of Puppis A generated from existing XMM-Newton and Chandra data. In this paper, we mainly focus on the RGS data, while we also utilize data taken by the European Photon Imaging Camera (EPIC: Turner et al. 2001; Strüder et al. 2001) to support our RGS analyses. The exposure times after removing intervals affected by soft protons are $13.5 \mathrm{ks}$ and $20.8 \mathrm{ks}$ for the NK and BEK, respectively. All the raw data are processed using version 11.0 .0 of the XMM Science Analysis Software and the latest calibration data files available at the time of the analysis.

Since the RGS is a slitless spectrometer, off-axis sources along the dispersion direction are detected at wavelength positions shifted with respect to the on-axis source. Spatial displacement of $1^{\prime}$ corresponds to a wavelength shift of $0.138 \AA$ (or $4 \mathrm{eV} /$ arcmin at $0.6 \mathrm{keV}$ ) for the first spectral order. Because the BEK and the NK are fairly compact $\left(2^{\prime}-3^{\prime}\right)$ features with much higher surface brightness than their surroundings, the RGS is capable of producing high-resolution spectra for them, with an order-of-magnitude better resolution than nondispersive CCDs. In fact, there are a number of successful RGS observations not only of moderately extended SNRs in the LMC/SMC (e.g., Rasmussen et al. 2001), but also of a bright knot along the northwestern rim of SN 1006 (Vink et al. 2003).

As shown in Fig. 11 we divide the eastern RGS field of view into four sectors spaced by $0^{\prime} .8$ along the crossdispersion axis, while we extract one spectrum from the $1^{\prime} .6$-width region for the north. In this way, we mitigate the photon number difference between the BEK and the NK. Also, slicing the BEK region allows us to generate more accurate RGS response files than the response file for the entire BEK region as we describe below. We smooth RGS responses originally designed for point sources, based on emission profiles along the dispersion direction with the rgsrmf smooth software. For the input to the software, we arrange the X-ray images such that Chandra covers regions around the BEK/NK while $X M M-N e w t o n$ covers the remaining regions, and we take account of vignetting effects of XMM-Newton's $\mathrm{X}$-ray telescope. We generate energy-dependent RGS responses using energy-band images in $0.35-0.4 \mathrm{keV}$, $0.4-0.5 \mathrm{keV}, 0.5-0.6 \mathrm{keV}, 0.6-0.7 \mathrm{keV}, 0.7-0.85 \mathrm{keV}, 0.85-$ $0.97 \mathrm{keV}, 0.97-1.2 \mathrm{keV}$, and $1.2-1.5 \mathrm{keV}$. Figure 1 right shows the X-ray emission profiles in $0.5-0.6 \mathrm{keV}$ for the five regions. These profiles, smoothed by the point spread function of the telescope, become the RGS responses themselves for the corresponding energy band. We note from this figure that the contribution from the $\mathrm{NK}$ in BEK spectra is almost negligible due to vignetting effects (the NK is slightly seen around $\mathrm{x}=33^{\prime}$ in the upper four panels in Fig. 1 right).

For RGS background (BG), we use a blank sky observation (Lockman hole: Obs.ID 0147511601) for which the RGS spectrum is free from apparent line emission. In addition, we need to subtract local BG, since the Vela SNR is superposed on Puppis A. To estimate local BG, we simulate RGS spectra based on plasma parameters derived by modeling EPIC spectra outside Puppis A. The simulated local BG is added to the blank sky spectrum, resulting in a total $\mathrm{BG}$. The total $\mathrm{BG}$ is dominated by local BG, and is less than $1 \%$ of the source emission. As for the EPIC BG, we use blank-sky data prepared by Read \& Ponman (2003). Since X-ray emission from Vela is much weaker than the BEK/NK features, especially in the higher energy band, we do not take account of local $\mathrm{BG}$ for the EPIC spectra.

Figure 2 shows BG-subtracted spectra for the five regions. To make the best use of XMM-Newton's capability, three different data sets are analyzed together: the first order RGS spectra below $0.6 \mathrm{keV}$, the second order RGS spectra in the $0.65-1.5 \mathrm{keV}$ band, and the MOS spectra above $1.1 \mathrm{keV}$. Note that the second or- 
der RGS spectra, which show the best spectral resolution among all the $\mathrm{X}$-ray spectrometers onboard $X M M$ Newton, do not cover the energy range below $0.65 \mathrm{keV}$, and that above $\sim 1 \mathrm{keV}$ the signal-to-noise ratio of the EPIC is much better than that of the RGS. We sum spectra from two co-aligned RGS spectrometers, RGS1 and RGS2, to improve photon statistics. We extract MOS spectra from the same sliced regions as the RGS, limiting around the BEK or the NK. As expected from previous X-ray observations, the RGS spectra are dominated by a number of emission lines, including $\mathrm{C} \operatorname{Ly} \alpha$ $@ 0.375 \mathrm{keV}, \mathrm{N} \mathrm{He} \alpha$ triplet @ $0.42 \mathrm{keV}, \mathrm{N} \mathrm{Ly} \alpha+\mathrm{N} \mathrm{He} \beta$ $@ 0.5 \mathrm{keV}, \mathrm{O}$ He $\alpha$ triplet @0.57 keV, O Ly $\alpha @ 0.654 \mathrm{keV}$, $\mathrm{O} \mathrm{He} \beta @ 0.666 \mathrm{keV}, \mathrm{Fe} \mathrm{L}(3 \mathrm{~s} \rightarrow 2 \mathrm{p})$ complex @ $0.73 \mathrm{keV}$, O Ly $\beta @ 0.775 \mathrm{keV}$, Fe L $(3 \mathrm{~d} \rightarrow 2 \mathrm{p})$ complex @ $0.83 \mathrm{keV}$, Ne He $\alpha$ triplet @ $0.92 \mathrm{keV}, \mathrm{Mg}$ He $\alpha$ triplet @ $1.35 \mathrm{keV}$, and Si He $\alpha$ triplet @1.85 keV. In particular, as shown in the right (and left) panels of Fig. 2, forbidden and resonance lines in the $\mathrm{He} \alpha$ triplet of $\mathrm{O}(\mathrm{N}$ and $\mathrm{Ne}$ as well) are clearly resolved for the first time for the two knots. We also note that this is the first clear detection of $\mathrm{C}$ and $\mathrm{N}$ lines from Puppis A.

We fit the spectra with an absorbed bremsstrahlung component plus 49 Gaussian components. Free parameters are the $\mathrm{H}$ column density $N_{\mathrm{H}}$, the electron temperature $k T_{\mathrm{e}}$, and the normalization of the bremsstrahlung component. We also allow normalizations of most Gaussians to vary freely. Since O Ly $\gamma$ and $\mathrm{O}$ Ly $\delta$ lines fall in the $\mathrm{Fe} \mathrm{L}$ line forest, we fix the normalization ratios of $\mathrm{O} \operatorname{Ly} \gamma / \mathrm{O} \operatorname{Ly} \beta$ and $\mathrm{O} \operatorname{Ly} \delta / \mathrm{O} \operatorname{Ly} \beta$ to 0.3 and 0.1 as are expected at $k T_{\mathrm{e}} \sim 0.5 \mathrm{keV}$ (Hwang et al. 2005). We note that the $k T_{\mathrm{e}}$ values of the bremsstrahlung component are mainly determined by continuum emission above $1.5 \mathrm{keV}$. Thus, fixing ratios of Gaussian normalizations does not affect the $k T_{\mathrm{e}}$ measurements. Line centers of the most prominent 21 Gaussians are left as free parameters, while those for $\mathrm{CHe} \beta$ and two $\mathrm{Fe} L$ lines ( $\mathrm{G}^{8}$ and D) are systematically shifted with respect to those of their neighboring lines ( $\mathrm{H}$ and $\mathrm{E}$ ). Other weak Gaussians' centers are fixed to the theoretically expected values (Smith et al. 2001). The widths of all Gaussians are fixed to zero, since significant broadening is not required from a statistical point of view; note that apparent line widths seen in the RGS spectra in Fig. 2 are due to spatial extent of the source as described in the second paragraph in this section. With this fitting strategy, we obtain fairly good fits for all the spectra as shown in Fig. 2 , The fit results are summarized in Table1, where we omit weak Gaussians.

The values of $N_{\mathrm{H}}$ are consistent with recent X-ray measurements (Hwang et al. 2005, 2008; Katsuda et al. 2010). The $k T_{\mathrm{e}}$ values are somewhat lower than previous results. However, prominent line intensities, which are essential for the discussion below, are not affected by the temperature difference, as the fraction of underlying continuum is very small. Line intensity ratios are basically reproduced by thermal emission models having plausible plasma parameters (i.e., electron temperature of $0.5 \mathrm{keV}$ and ionization timescale of $10^{11} \mathrm{~cm}^{-3} \mathrm{~s}$ : Hwang et al. 2005).

On the other hand, as summarized in Table 2, the

8 We use the same labeling for Fe XVII L lines as in Gillaspy et al. (2011). intensity ratios of forbidden line to resonance line in He $\alpha$ triplets (hereafter, F/R ratios) are inconsistent with model predictions, for which we assume a planeparallel shock model with a distribution of ionization timescales appropriate for a plane-parallel shock (vpshock model in conjunction with augmented NEI version 2.0: Borkowski et al. 2001); this emission model is often used to describe X-ray spectra from Puppis A. In calculating the model $\mathrm{F} / \mathrm{R}$ ratio, we take a reasonable range of plasma conditions in the BEK $\left(k T_{\mathrm{e}}=\right.$ $0.3-0.7 \mathrm{keV}$ and $n_{\mathrm{e}} t=10^{10}-5 \times 10^{11} \mathrm{~cm}^{-3} \mathrm{~s}$ : Hwang et al. 2005), given that the RGS spectra are integrated along the dispersion direction as well as the line of sight. From Table 2, we see that the F/R-ratio anomaly for $\mathrm{O}$ and $\mathrm{Ne}$ ions is particularly evident in the E2-E4 regions compared with that in the E1 and NK regions, while the $\mathrm{F} / \mathrm{R}$ ratios for $\mathrm{N}$ ions are marginally consistent among the five regions. The discrepancy can not be solved by multiple plasma components, since the model predictions already consider various (reasonable) plasma conditions. Although the ratios could be reproduced at a very low $n_{\mathrm{e}} t$ value of $\sim 10^{9} \mathrm{~cm}^{-3} \mathrm{~s}$, in such a low ionization condition, no Ne-like Fe L lines nor H-like Ne lines can be emitted, which is in stark contrast to the observed X-ray spectra. Thus, the $\mathrm{F} / \mathrm{R}$ ratios cannot be reproduced by thermal emission models.

We next investigate whether the anomalous $\mathrm{F} / \mathrm{R}$ ratio is due to reduction of the resonance line or increase of the forbidden line, by comparing the observed line intensities with model expectations. To this end, we fit the X-ray spectra (in the E2 region) with an absorbed vpshock model, excluding the particularly anomalous $\mathrm{O} \mathrm{He} \alpha$ triplets. After fitting, we recover the $\mathrm{O} \mathrm{He} \alpha$ triplet lines to compare the data with the model. We find that the intensity of the forbidden line inferred from the vpshock model is weaker than the data and that the model intensity of the resonance line is stronger. This result suggests both reduction of the resonance line and increase of the forbidden line. However, we need to be careful about limitations of our spectral modeling. This is because the X-ray emission other than the $\mathrm{O}$ He $\alpha$ triplets may also not be interpreted in the frame of pure thermal emission models, due to contamination of CX emission, effects of resonance line scattering, or some other processes. If this is the case, the plasma parameters including $\mathrm{O}$ abundance, the electron temperature, and the ionization timescale inferred by our thermal emission modeling should have some systematic uncertainties, leading to an incorrect model intensity of the $\mathrm{O}$ He $\alpha$ triplet. Therefore, it is difficult to infer the model $\mathrm{O}$ He $\alpha$ intensity accurately enough at this point. We will revisit this issue in our future work that will include developments of more sophisticated emission models.

\section{DISCUSSIONS}

We have presented high-resolution X-ray spectra of the BEK/NK features in the Galactic SNR Puppis A. The forbidden and resonance lines in the $\mathrm{He} \alpha$ triplets are clearly resolved, and their intensity ratios $(\mathrm{F} / \mathrm{R})$ are found to be generally higher than predictions from thermal plasma models. This anomaly is particularly evident in the BEK, while the RGS spectrum of the NK as well as the Einstein FPCS spectra of the northeastern portion (Winkler et al. 1981a b) are closer to predictions of 

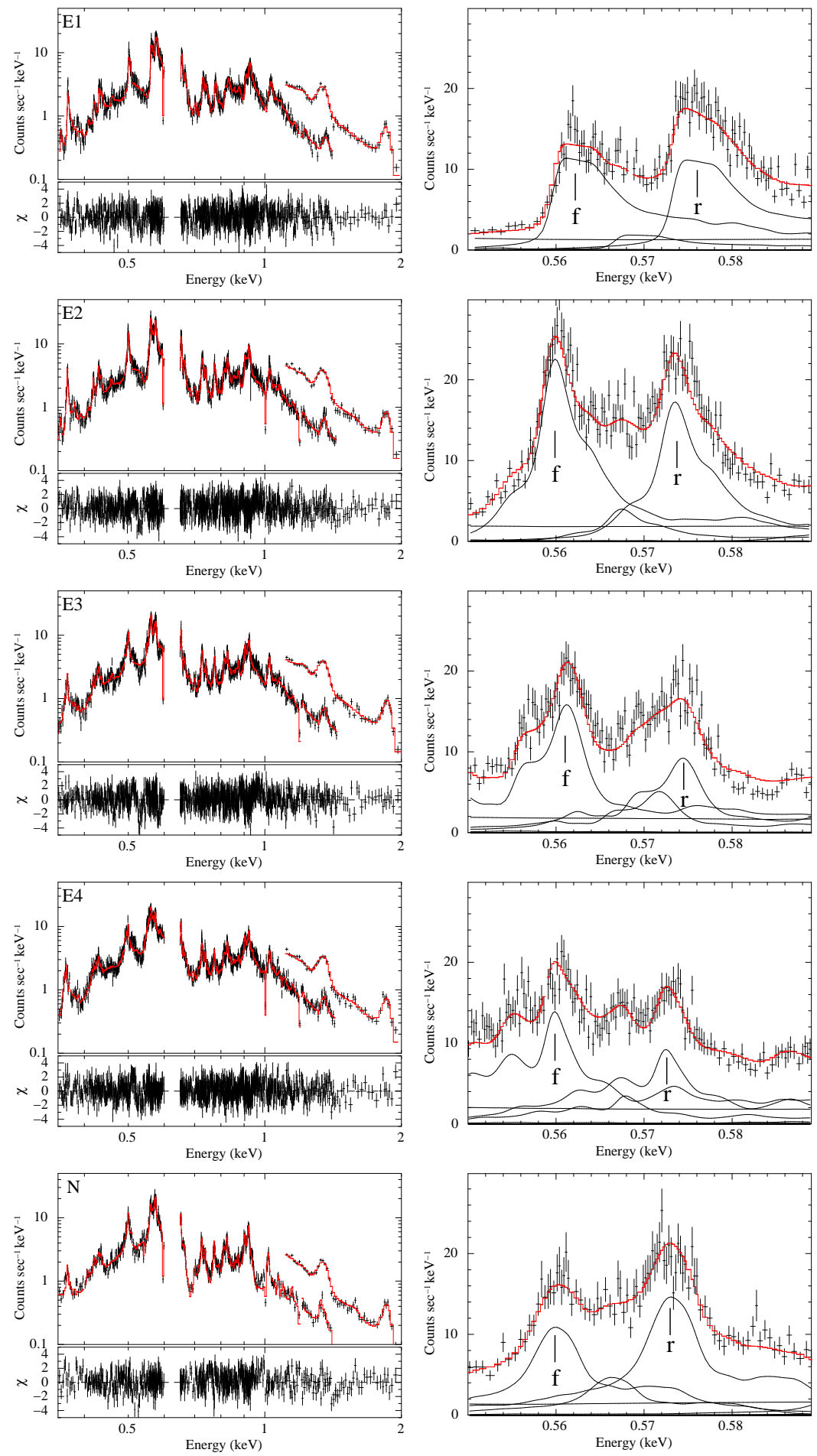

FIG. 2.- Left: $X M M-N e w t o n$ spectra of the five regions in Fig. 1 The data covering below $0.6 \mathrm{keV}, 0.65-1.5 \mathrm{keV}$, and above $1.1 \mathrm{keV}$ are the first order RGS1+2, the second order RGS1+2, and MOS1+2, respectively. These data are simultaneously fitted with a phenomenological model (see, text), and the best-fit models are shown in red. Lower panels show the residuals. Right: Close-up RGS spectra for O He $\alpha$ triplets with individual best-fit model components. Forbidden and resonance lines are indicated as "f" and "r", respectively. 
thermal models.

There are two ways to enhance the $\mathrm{F} / \mathrm{R}$ ratio: $(1)$ an increase of the forbidden line flux or (2) a reduction of the resonance line flux. Possible mechanisms for the former are either $\mathrm{H}$-like $\rightarrow$ He-like recombination/CX processes or inner-shell ionization of Li-like ions due to the higher statistical weight. As for the latter, we consider resonance-scattering effects; only resonance lines are scattered out of the line of sight because of their higher optical depths. Among several SNRs for which highresolution spectroscopy has been performed, anomalous $\mathrm{F} / \mathrm{R}$ ratios have only been reported in two LMC SNRs, DEM L71 (van der Hevden et al. 2003) and 0506-68 a.k.a N23 (Broersen et al. 2011). In these cases, the authors attributed the anomaly to recombination and/or resonance scattering, paying little or no attention to $\mathrm{CX}$. Below we discuss which mechanism mainly works for the case of Puppis A.

Effects of resonance scattering can be evaluated for each emission line from its optical depth, $\tau$ (e.g., Kaastra \& Mewe 1995). We calculate the value of $\tau$ using the BEK's typical plasma parameters; i.e., $\sim 0.5$ solar abundances, $k T_{\mathrm{e}} \sim 0.5 \mathrm{keV}, n_{\mathrm{e}} t \sim 10^{11} \mathrm{~cm}^{-3} \mathrm{~s}$, and the hydrogen column density of the X-ray-emitting plasma $\sim 2.4 \times 10^{19} \mathrm{~cm}^{-2}$ from $n_{\mathrm{e}} \sim 4 \mathrm{~cm}^{-3}$ (Arendt et al. 2010) multiplied by an assumed plasma depth of $3.2 \mathrm{pc}$, which corresponds to the $3^{\prime}$ size of the BEK at a distance of $2.2 \mathrm{kpc}$ (Reynoso et al. 2003, we refer to this distance hereafter). To derive oscillator strengths and ion fractions, we utilize the data bases from CHIANTI (Dere et al. 2009) and SPEX (specifically, the neij code: Kaastra, et al. 1996), respectively. Then, assuming no microturbulence, we calculate $\tau$ and the escape probability, $p$. The escape probabilities for resonance lines in He $\alpha$ triplets of $\mathrm{N}, \mathrm{O}$, and $\mathrm{Ne}$ are obtained to be 1, 0.92, and 0.92 , respectively, while those for forbidden lines are all unity because of their very small oscillator strengths. Therefore, we find that effects of resonance line scattering likely play a modest role, if any, in explaining the observed $\mathrm{F} / \mathrm{R}$ ratios for $\mathrm{N}$ and $\mathrm{O}$ in all the five regions, while the $\mathrm{F} / \mathrm{R}$ anomaly for Ne could be explained by the effects. Whereas optical depths could differ with changing the assumed plasma depth and microturbulence velocity, the difference seems small in our case. For example, in order to reproduce the observed $\mathrm{F} / \mathrm{R}$ ratio in the E3 region, we need a plasma depth of $77 \mathrm{pc}$ (or $2^{\circ}$ ) which is obviously too long for this region. Also, a microturbulence velocity of $100 \mathrm{~km} \mathrm{~s}^{-1}$ (which is statistically allowed by our RGS spectral analysis) would reduce $\tau$ to $\sim 0.12$ and increase $p$ to $\sim 0.95$ for a reasonable plasma depth of $3.2 \mathrm{pc}$. It should be noted that the Fe $\mathrm{L}$ line ratio, $(\mathrm{F}+\mathrm{G}+\mathrm{H}) / \mathrm{C}$, should be more sensitive to resonance scattering than the $\mathrm{O}$ VII $\mathrm{F} / \mathrm{R}$ ratio: we expect $\tau \sim 0.02$ and $p \sim 0.99$ for $\mathrm{F}+\mathrm{G}+\mathrm{H}$ and $\tau \sim 0.43$ and $p \sim 0.84$ for $\mathrm{C}$ for the plasma conditions in the BEK. We find in Table 2 that the observed $(\mathrm{F}+\mathrm{G}+\mathrm{H}) / \mathrm{C}$ ratios are indeed slightly higher than those of the vpshock prediction. While the measured line ratios suggest the presence of resonance scattering for the Fe L lines, the degree of the effect is not as significant as what is expected from the anomaly of $\mathrm{O}$ VII $\mathrm{F} / \mathrm{R}$ ratios. Therefore, we are required to examine other possibilities.

X-ray-emitting recombining plasmas have been recently found in several SNRs (e.g., Yamaguchi et al. 2009; Uchida et al. 2012, and references therein). The recombining plasma is characterized by strong radiative recombination continua (RRC) and enhanced Ly $\alpha / \mathrm{He} \alpha$ ratios. We find that signatures of RRCs (i.e., strong recombination edges) are not evident in the RGS and MOS spectra in Fig. 2 (and also Suzaku XIS spectra which are not shown in this paper), although the Fe L and/or other line emission might make it difficult for us to detect such signatures. Also, the $\mathrm{Ly} \alpha / \mathrm{He} \alpha$ ratios for $\mathrm{N}, \mathrm{O}$, and $\mathrm{Ne}$ in the five regions are all well within thermal predictions as can be seen in Table 2, while the BEK regions show somewhat larger ratios than those in the NK. In addition, recombination processes would enhance the $\mathrm{Fe}$ $\mathrm{L}$ line ratio $(\mathrm{F}+\mathrm{G}+\mathrm{H}) /(\mathrm{C}+\mathrm{D}+\mathrm{E})$ to $>25$ (Liedahl et al. 1990), which is inconsistent with our RGS measurements of 1.2-2.4 in the BEK and the NK. Furthermore, our spectral fitting of the combined RGS and MOS spectra with a recombining plasma model (the cie model in SPEX: Kaastra, et al. 1996) failed to reproduce the entire X-ray spectrum; the model requires too low an electron temperature to explain emission above $1 \mathrm{keV}$. These investigations indicate that the plasma here is not recombining.

Signatures of inner-shell ionization processes of Lilike ions can be found as Li-like satellite lines as well as an enhanced forbidden-to-intercombination (F/I) ratio compared with collisionally excited emission (e.g., Porquet et al. 2010). In the RGS spectra, there is no indication of satellite lines, however. Also, the measured $\mathrm{F} / \mathrm{I}$ ratios of $\mathrm{He} \alpha$ triplets in Table 2 are all marginally consistent with thermal expectations; we need to take account of considerable systematic uncertainties on the intensity of the intercombination line due to its weakness compared to the surrounding forbidden and resonance lines. These facts led us to conclude that inner-shell ionization processes are not working efficiently in this region. We note that, whereas line emission from Li-like $\mathrm{O}$ is seen in the far-ultraviolet spectrum of the BEK (Blair, et al. 1995), the abundance of Li-like $\mathrm{O}$ would be small in the $\mathrm{X}$-ray-emitting region.

We next assess the feasibility of the CX scenario by calculating the expected CX flux, following Lallement (2004). The volume emissivity of CX is expressed as $P_{\mathrm{CX}}=\sigma_{\mathrm{CX}} n_{\mathrm{H}} n_{\mathrm{i}} V_{\mathrm{r}}$. We let $\sigma_{\mathrm{CX}}$ be the CX cross section between neutral $\mathrm{H}$ and ions of interest, $n_{\mathrm{H}}$ the neutral $\mathrm{H}$ density, $n_{\mathrm{i}}$ the ion density, and $V_{\mathrm{r}}$ the relative $\mathrm{H}$-ion velocity. We first consider the O VII forbidden line, so that $\sigma_{\mathrm{CX}}$ is $3.3 \times 10^{-15} \mathrm{~cm}^{-2}$ (Bodewits, et al. 2007). The value of $n_{\mathrm{H}}$ can be taken from the density of cold/warm clouds immersed in X-ray-emitting plasma, which is $\sim 50 \mathrm{~cm}^{-3}$ (Teske \& Petre 1987). The value of $n_{\mathrm{i}}$ is the density of O VIII in the hot plasma, which is $\sim 2 \times 10^{-4} \mathrm{~cm}^{-3}$ for the BEK (Hwang et al. 2008; Arendt et al. 2010). We assume that $V_{\mathrm{r}}=500 \mathrm{~km} \mathrm{~s}^{-1}$, roughly the shock velocity that can produce the X-rayemitting plasma in the BEK. These parameters give $P_{\mathrm{CX}} \sim 1.7 \times 10^{-9}$ photons $\mathrm{cm}^{-3} \mathrm{~s}^{-1}$. The CX-emitting vol- 
TABLE 1

SPECTRAL-FIT PARAMETERS

\begin{tabular}{|c|c|c|c|c|c|c|c|}
\hline \multirow{2}{*}{\multicolumn{2}{|c|}{ Component }} & \multirow[t]{2}{*}{ Parameter } & \multicolumn{5}{|c|}{ Region } \\
\hline & & & E1 & $\mathrm{E} 2$ & E3 & E4 & $\mathrm{N}$ \\
\hline \multirow{3}{*}{\multicolumn{2}{|c|}{$\begin{array}{l}\text { Absorption } \\
\text { Bremsstrahlung }\end{array}$}} & $N_{\mathrm{H}}\left(10^{21} \mathrm{~cm}^{-2}\right)$ & $2.70 \pm 0.01$ & $2.80 \pm 0.01$ & $2.80 \pm 0.01$ & $2.85 \pm 0.01$ & $2.58 \pm 0.01$ \\
\hline & & $k T_{\mathrm{e}}(\mathrm{keV})$ & $0.32 \pm 0.01$ & $0.31 \pm 0.01$ & $0.33 \pm 0.01$ & $0.32 \pm 0.01$ & $0.30 \pm 0.01$ \\
\hline & & Normalization $^{a}$ & $5264 \pm 93$ & $9124 \pm 133$ & $6744 \pm 117$ & $7808 \pm 117$ & $5397 \pm 90$ \\
\hline \multirow{2}{*}{\multicolumn{2}{|c|}{ Gaussian: $\mathrm{C}$ Ly $\alpha+\mathrm{Si} \mathrm{L}+\mathrm{S} \mathrm{L}$}} & Center $(\mathrm{eV})$ & $366.8_{-0.1}^{+0.2}$ & $367.2_{-0.1}^{+0.2}$ & $366.7_{-0.3}^{+0.4}$ & $365.4_{-0.3}^{+0.4}$ & $367.4_{-0.8}^{+0.3}$ \\
\hline & & Normalization $^{a}$ & $822 \pm 80$ & $1486 \pm 118$ & $1257 \pm 139$ & $1723 \pm 139$ & $380 \pm 52^{\circ}$ \\
\hline \multirow{2}{*}{\multicolumn{2}{|c|}{$\mathrm{N} \operatorname{He} \alpha(\mathrm{f})$}} & Center $(\mathrm{eV})$ & $419.2_{-0.5}^{+0.2}$ & $419.2 \pm 0.3$ & $418.4 \pm 0.5$ & $419.7 \pm 0.5$ & $420.5_{-0.6}^{+0.8}$ \\
\hline & & Normalization $^{a}$ & $100 \pm 18$ & $201 \pm 26$ & $145 \pm 26$ & $243 \pm 33$ & $82 \pm 16$ \\
\hline \multirow{2}{*}{\multicolumn{2}{|c|}{$\mathrm{N} \operatorname{He} \alpha(\mathrm{i})$}} & Center $(\mathrm{eV})$ & $426^{b}$ & $426^{b}$ & $426^{b}$ & $426^{b}$ & $426^{b}$ \\
\hline & & Normalization $^{a}$ & $<15$ & $57 \pm 23$ & $72 \pm 24$ & $<57$ & $18 \pm 15$ \\
\hline \multirow{2}{*}{\multicolumn{2}{|c|}{$\mathrm{N} \operatorname{He} \alpha(\mathrm{r})$}} & Center $(\mathrm{eV})$ & $429.7_{-0.2}^{+0.3}$ & $430.1_{-0.2}^{+0.3}$ & $429.9 \pm 0.5$ & $429.7 \pm 0.5$ & $430.6 \pm 0.5$ \\
\hline & & Normalization $^{a}$ & $146 \pm 17$ & $223 \pm 24$ & $169 \pm 23$ & $199 \pm 27$ & $72 \pm 15$ \\
\hline \multirow{2}{*}{\multicolumn{2}{|c|}{$\mathrm{C} \operatorname{Ly} \beta$}} & Center $(\mathrm{eV})$ & $434.9_{-0.2}^{+0.7}$ & $435.1_{-0.3}^{+0.6}$ & $435.2_{-0.6}^{+0.7}$ & $434.7_{-0.6}^{+0.7}$ & $435.2_{-0.6}^{+0.7}$ \\
\hline & & Normalization $^{a}$ & $60 \pm 14$ & $87 \pm 18$ & $55 \pm 18$ & $87 \pm 22$ & $51 \pm 13$ \\
\hline \multirow{2}{*}{\multicolumn{2}{|c|}{$\mathrm{C} \mathrm{Ly} \gamma$}} & Center $(\mathrm{eV})$ & $459^{b}$ & $459^{b}$ & $459^{b}$ & $459^{b}$ & $459^{b}$ \\
\hline & & Normalization $^{a}$ & $25 \pm 8$ & $31 \pm 10$ & $24 \pm 10$ & $14 \pm 12$ & $<8$ \\
\hline \multirow{2}{*}{\multicolumn{2}{|c|}{$\mathrm{N} \mathrm{Ly} \alpha+\operatorname{He} \beta$}} & Center $(\mathrm{eV})$ & $499.4 \pm 0.2$ & $500.2_{-0.2}^{+0}$ & $499.8_{-0.3}^{+0.2}$ & $499.6_{-0.3}^{+0.2}$ & $499.6_{-0.4}^{+0.5}$ \\
\hline & & Normalization $^{a}$ & $143 \pm 9$ & $254 \pm 13$ & $222 \pm 12$ & $252 \pm 14$ & $162 \pm 11^{4}$ \\
\hline \multirow{2}{*}{\multicolumn{2}{|c|}{$\mathrm{O} \operatorname{He} \alpha(\mathrm{f})$}} & Center $(\mathrm{eV})$ & $559.8_{-0.4}^{+0.4}$ & $560.7 \pm 0.1$ & $560.5 \pm 0.5$ & $560.2 \pm 0.5$ & $561.2 \pm 0.2$ \\
\hline & & Normalization $^{a}$ & $705 \pm 27$ & $1167 \pm 36$ & $1042 \pm 36$ & $1119 \pm 38$ & $417 \pm 23$ \\
\hline \multirow{2}{*}{\multicolumn{2}{|c|}{$\mathrm{O} \operatorname{He} \alpha(\mathrm{i})$}} & Center $(\mathrm{eV})$ & $566.8_{-0.9}^{+1.0}$ & $568.3_{-1.1}^{+0.3}$ & $570.7_{-0.7}^{+0.4}$ & $568.5_{-0.7}^{+0.4}$ & $567.5_{-1.1}^{+0.4}$ \\
\hline & & Normalization $^{a}$ & $107 \pm 24$ & $190 \pm 30$ & $298 \pm 31$ & $255 \pm 34$ & $168 \pm 22$ \\
\hline \multirow{2}{*}{\multicolumn{2}{|c|}{$\mathrm{OHe} \alpha(\mathrm{r})$}} & Center $(\mathrm{eV})$ & $573.1 \pm 0.2$ & $574.1_{-0.2}^{+0.3}$ & $573.4 \pm 0.1$ & $572.9 \pm 0.1$ & $574.3_{-0.3}^{+0.2}$ \\
\hline & & Normalization $^{a}$ & $601 \pm 25$ & $771 \pm 30$ & $527 \pm 31$ & $646 \pm 33$ & $492 \pm 21$ \\
\hline \multirow{2}{*}{\multicolumn{2}{|c|}{$\mathrm{O} \mathrm{Ly} \alpha$}} & Center $(\mathrm{eV})$ & $652.7_{-0.1}^{+0.5}$ & $653.4 \pm 0.2$ & $653.0 \pm 0.2$ & $652.8 \pm 0.2$ & $653.4 \pm 0.4$ \\
\hline & & Normalization $^{a}$ & $979 \pm 38$ & $1223 \pm 47$ & $1154 \pm 46$ & $1329 \pm 51$ & $631 \pm 36$ \\
\hline \multirow{2}{*}{\multicolumn{2}{|c|}{$\mathrm{OHe} \beta$}} & Center $(\mathrm{eV})$ & $664.4 \pm 0.8$ & $665.0_{-0.7}^{+0.3}$ & $665.4_{-1.4}^{+1.3}$ & $666.0_{-1.4}^{+1.3}$ & $667.0 \pm 1$ \\
\hline & & Normalization $^{a}$ & $161 \pm 24$ & $285 \pm 29$ & $197 \pm 27$ & $\begin{array}{l}136 \pm 29 \\
\end{array}$ & $110 \pm 17$ \\
\hline \multirow{2}{*}{\multicolumn{2}{|c|}{$\mathrm{OHe} \gamma$}} & Center $(\mathrm{eV})$ & $699.0_{-0.8}^{+0.9}$ & $700.7 \pm 0.6$ & $698.9_{-0.8}^{+1.1}$ & $698.2_{-0.8}^{+1.1}$ & $700.7 \pm 1.1$ \\
\hline & & Normalization $^{a}$ & $68 \pm 8$ & $74 \pm 10$ & $65 \pm 11$ & $74 \pm 13^{\circ}$ & $42 \pm 9$ \\
\hline \multirow{2}{*}{\multicolumn{2}{|c|}{$\mathrm{Fe} \mathrm{L}(\mathrm{G}+\mathrm{H})$}} & Center $^{c}(\mathrm{eV})$ & $724.9_{-0.1}^{+0.4}$ & $725.1_{-0.4}^{+0.2}$ & $724.9_{-0.3}^{+0.1}$ & $725.2_{-0.3}^{+0.1}$ & $725.2_{-0.4}^{+0.1}$ \\
\hline & & Normalization $^{a}$ & $247_{-15}^{+16}$ & $\begin{array}{l}362_{-20}^{+21} \\
-4\end{array}$ & $331_{-18}^{+19}$ & $332_{-19}^{+20}$ & $156_{-12}^{+13}$ \\
\hline \multirow{2}{*}{\multicolumn{2}{|c|}{$\mathrm{Fe} L(\mathrm{~F})$}} & Center $(\mathrm{eV})$ & $739.3_{-0.5}^{+0.8}$ & $738.5_{-0.7}^{+0.1}$ & $739.3_{-0.3}^{+0.9}$ & $738.1_{-0.3}^{+0.9}$ & $738.9_{-0.3}^{+0.8}$ \\
\hline & & Normalization $^{a}$ & $89 \pm 11$ & $156 \pm 13$ & $94 \pm 12$ & $99 \pm 12$ & $65 \pm 8$ \\
\hline \multirow{2}{*}{\multicolumn{2}{|c|}{$\mathrm{O} \operatorname{Ly} \beta$}} & Center $(\mathrm{eV})$ & $773.5_{-0.6}^{+0.2}$ & $773.8 \pm 0.3$ & $773.3_{-0.6}^{+0.4}$ & $772.9_{-0.6}^{+0.4}$ & $773.7 \pm 0.5$ \\
\hline & & Normalization $^{a}$ & $151 \pm 7$ & $187 \pm 8$ & $160 \pm 7$ & $169 \pm 8$ & $97 \pm 6$ \\
\hline & $\mathrm{Fe} \mathrm{L}(\mathrm{D}+\mathrm{E})$ & Center $^{c}(\mathrm{eV})$ & $810.6 \pm 0.8$ & $810.1 \pm 0.2$ & $810.0 \pm 0.4$ & $810.0 \pm 0.4$ & $810.1 \pm 0.7$ \\
\hline & & Normalization $^{a}$ & $74_{-9}^{+10}$ & $110_{-10}^{+11}$ & $98_{-10}^{+11}$ & $90_{-10}^{+11}$ & $50_{-6}^{+7}$ \\
\hline & $\mathrm{Fe} \mathrm{L}(\mathrm{C})$ & Center $(\mathrm{eV})$ & $826.6_{-1.0}^{+0.4}$ & $826.1 \pm 0.5$ & $825.6 \pm 0.5$ & $826.1 \pm 0.5$ & $826.2_{-0.6}^{+0.3}$ \\
\hline & & Normalization $^{a}$ & $105 \pm 6$ & $173 \pm 0.8$ & $135 \pm 7$ & $152 \pm 8$ & $78 \pm 5$ \\
\hline & $\mathrm{Ne} \operatorname{He} \alpha(\mathrm{f})+\mathrm{Fe} \mathrm{L}$ & Center $(\mathrm{eV})$ & $902.0 \pm 0.6$ & $903.3_{-0.2}^{+0.5}$ & $902.6_{-0.2}^{+0.4}$ & $902.6_{-0.2}^{+0.4}$ & $903.5_{-0.4}^{+0.6}$ \\
\hline & & Normalization $^{a}$ & $122 \pm 6$ & $185 \pm 7$ & $156 \pm 6$ & $175 \pm 7$ & $75 \pm 4$ \\
\hline & $\mathrm{Ne} \operatorname{He} \alpha(\mathrm{i})+\mathrm{Fe} \mathrm{L}$ & Center $(\mathrm{eV})$ & $915^{b}$ & $915^{b}$ & $915^{b}$ & $915^{b}$ & $915^{b}$ \\
\hline & & Neormalization $^{a}$ & $63 \pm 6$ & $53 \pm 7$ & $68 \pm 6$ & $50 \pm 7$ & $18 \pm 4$ \\
\hline & $\mathrm{Ne} \operatorname{He} \alpha(\mathrm{r})+\mathrm{Fe} \mathrm{L}$ & Center $(\mathrm{eV})$ & $921.2_{-0.5}^{+0.3}$ & $921.6_{-0.3}^{+0.1}$ & $921.2_{-0.4}^{+0.3}$ & $921.8_{-0.4}^{+0.3}$ & $922.4 \pm 0.2$ \\
\hline & & Normalization $^{a}$ & $141 \pm 6$ & $224 \pm 7$ & $168 \pm 6$ & $188 \pm 7$ & $111 \pm 5$ \\
\hline & $\operatorname{Ne} \mathrm{Ly} \alpha+\mathrm{Fe} \mathrm{L}$ & Center $(\mathrm{eV})$ & $1023.6_{-1.4}^{+1.2}$ & $1022.5_{-1.2}^{+0.5}$ & $1020.5_{-1.4}^{+0.2}$ & $1022.1_{-1.4}^{+0.2}$ & $1020.4_{-0.8}^{+1.1}$ \\
\hline & & Normalization $^{a}$ & $83 \pm \overline{5}^{1.4}$ & $97 \pm \overline{6}^{1.2}$ & $96 \pm \overline{5}^{1.4}$ & $102 \pm 6$ & $35 \pm 3^{-0.8}$ \\
\hline$\chi^{2} /$ d.o.f. & & & $1423 / 968$ & $1740 / 1151$ & $1698 / 1114$ & $1664 / 1181$ & $949 / 612$ \\
\hline
\end{tabular}

Note. - ${ }^{a}$ In units of $10^{-4}$ photons $\mathrm{cm}^{-2} \mathrm{~s}^{-1}$.

${ }^{b}$ Fixed values.

${ }^{c}$ Line centers of $\mathrm{H}$ and $\mathrm{E}$ are shown, while those of $\mathrm{G}$ and $\mathrm{D}$ are systematically shifted by $+2 \mathrm{eV}$ from $\mathrm{H}$ and $\mathrm{E}$, respectively

ume is calculated by the thickness of the CX layer, $l_{\mathrm{CX}}$, times the interface area between the clouds and the hot plasma. The value of $l_{\mathrm{CX}}$ is equated to the mean free path for $\mathrm{H}$-proton $\mathrm{CX}$. Thus, $l_{\mathrm{CX}}$ is of the order of $1 / \sigma n_{\mathrm{p}}$, with $\sigma$ being the $\mathrm{H}$-proton $\mathrm{CX}$ cross section $\left(10^{-15} \mathrm{~cm}^{-2}\right.$ : McClure 1966) and $n_{\mathrm{p}}$ being the proton density in the plasma $\left(4 \mathrm{~cm}^{-3}\right.$ : Arendt et al. 2010). Considering that only $\sim 30 \%$ neutral $\mathrm{H}$ can charge-exchange before getting collisionally ionized (Lallement 2004) for the BEK's plasma condition, we obtain an effective CX thickness of $\sim 7.5 \times 10^{13} \mathrm{~cm}$. An assumed cylindrical interface with a diameter of $3^{\prime}$ would give a surface area of the CX layer of $\sim 3 \times 10^{37} \mathrm{~cm}^{2}$ for each region, resulting in a CX-emitting volume of $\sim 2.3 \times 10^{51} \mathrm{~cm}^{3}$. With $P_{\mathrm{CX}}$ and the emitting volume estimated, and an assumption of a uniform $P_{\mathrm{CX}}$, we obtain an unabsorbed CX flux for the O VII forbidden line of $\sim 6 \times 10^{-3}$ photons $\mathrm{cm}^{-2} \mathrm{~s}^{-1}$ for each region in the BEK. Next, we repeat the calculation for forbidden lines of He-like $\mathrm{N}$ and $\mathrm{Ne}$. Using the relation that $\sigma_{\mathrm{CX}}$ is proportional to the atomic 
TABLE 2

LINE INTENSITY RATIOS

\begin{tabular}{|c|c|c|c|c|c|c|}
\hline \multirow[t]{2}{*}{ Element } & \multirow[t]{2}{*}{ Thermal predictions $^{a}$} & \multicolumn{5}{|c|}{ Region } \\
\hline & & E1 & E2 & E3 & $\mathrm{E} 4$ & $\mathrm{~N}$ \\
\hline $\mathrm{NF} / \mathrm{R}$ & $0.38-0.52$ & $0.68 \pm 0.15$ & $0.90 \pm 0.15$ & $0.86 \pm 0.19$ & $1.22 \pm 0.23$ & $1.14 \pm 0.33$ \\
\hline $\mathrm{O} F / \mathrm{R}$ & $0.42-0.56$ & $1.17 \pm 0.07$ & $1.51 \pm 0.08$ & $1.98 \pm 0.13$ & $1.73 \pm 0.11$ & $0.85 \pm 0.06$ \\
\hline $\mathrm{Ne}^{b} \mathrm{~F} / \mathrm{R}$ & $0.44-0.71$ & $0.87 \pm 0.06$ & $0.83 \pm 0.04$ & $0.93 \pm 0.05$ & $0.93 \pm 0.05$ & $0.68 \pm 0.05$ \\
\hline $\mathrm{N} \mathrm{F} / \mathrm{I}$ & $5.8-6.4$ & $>7.9$ & $3.49 \pm 1.46$ & $2.02 \pm 0.76$ & $>3.7$ & $4.48 \pm 3.73$ \\
\hline $\mathrm{O} F / \mathrm{I}$ & $4.2-5.1$ & $6.58 \pm 1.49$ & $6.14 \pm 0.99$ & $3.49 \pm 0.38$ & $4.39 \pm 0.60$ & $2.48 \pm 0.35$ \\
\hline $\mathrm{Ne}^{b} \mathrm{~F} / \mathrm{I}$ & $3.3-4.9$ & $1.92 \pm 0.20$ & $3.45 \pm 0.47$ & $2.30 \pm 0.22$ & $3.46 \pm 0.50$ & $4.01 \pm 0.87$ \\
\hline$(\mathrm{N} \operatorname{Ly} \alpha+\mathrm{N} \operatorname{He} \beta) / \mathrm{N} \operatorname{He} \alpha$ & $0.12-0.9$ & $0.58 \pm 0.07$ & $0.53 \pm 0.05$ & $0.58 \pm 0.06$ & $0.54 \pm 0.06$ & $0.94 \pm 0.13$ \\
\hline $\mathrm{O} \operatorname{Ly} \alpha / \mathrm{O} \mathrm{He} \alpha$ & $0.01-1.03$ & $0.69 \pm 0.03$ & $0.57 \pm 0.03$ & $0.62 \pm 0.03$ & $0.66 \pm 0.03$ & $0.59 \pm 0.04$ \\
\hline $\mathrm{Ne}^{b} \operatorname{Ly} \alpha / \mathrm{Ne}^{b} \mathrm{He} \alpha$ & $0-0.83$ & $0.25 \pm 0.02$ & $0.21 \pm 0.01$ & $0.24 \pm 0.01$ & $0.25 \pm 0.02$ & $0.17 \pm 0.02$ \\
\hline $\mathrm{Fe} L(\mathrm{~F}+\mathrm{G}+\mathrm{H}) / \mathrm{C}$ & $1.4-2.6$ & $3.19 \pm 0.26$ & $2.98 \pm 0.14$ & $3.15 \pm 0.23$ & $2.84 \pm 0.21$ & $2.83 \pm 0.26$ \\
\hline
\end{tabular}

number, $\mathrm{Z}$, and the ion number densities of $n_{\mathrm{NVI}} \sim$ $8 \times 10^{-6} \mathrm{~cm}^{-3}$ and $n_{\text {NeIX }} \sim 4 \times 10^{-5} \mathrm{~cm}^{-3}$ (which are based on plasma parameters in Hwang et al. 2005), we estimate the unabsorbed CX fluxes of the N VI and $\mathrm{Ne}$ IX forbidden lines to be $\sim 3 \times 10^{-4}$ photons $\mathrm{cm}^{-2} \mathrm{~s}^{-1}$ and $\sim 2 \times 10^{-3}$ photons $\mathrm{cm}^{-2} \mathrm{~s}^{-1}$, respectively.

We should keep in mind that the uncertainty of the CX flux estimate is fairly large. For example, we implicitly assume that hydrogen in the cold/warm clouds is entirely neutral due to rapid recombination caused by radiative cooling in the dense cloud. The hydrogen must be partially ionized, however, resulting in a lower CX flux. On the other hand, hot neutral hydrogen formed after H-proton CX reactions, dust destruction, and unshocked cold neutrals going through the (collisionless) shock would all provide additional neutrals in the hot plasma, increasing the CX flux. Also, the area of the interface region is quite uncertain. These factors suggest that our CX flux estimate should only be considered accurate to an order of magnitude.

To compare the expected CX flux with our measurements in Table 1, we need to correct for the spatial-integration factor. This is because the expected flux is estimated for the BEK feature, whereas the fluxes in Table 1 are integrated along the RGS dispersion direction. To estimate the correction (reduction) factors, we extract EPIC spectra from 3 '-long regions including the BEK, and normalize the RGS flux to equalize the EPIC flux in each region. In this way, the correction factors are derived to be 3.7-4.5 for the four BEK regions. The fluxes of the $\mathrm{N}, \mathrm{O}$, and $\mathrm{Ne} \mathrm{He} \alpha$ forbidden lines are, respectively, derived to be $\sim 5 \times 10^{-3}$ photons $\mathrm{cm}^{-2} \mathrm{~s}^{-1}$, $\sim 3 \times 10^{-2}$ photons $\mathrm{cm}^{-2} \mathrm{~s}^{-1}$,

and $\sim 5 \times 10^{-3}$ photons $\mathrm{cm}^{-2} \mathrm{~s}^{-1}$ for each region in the BEK. We thus find order-of-magnitude agreement with the expected CX fluxes. Given the considerable uncertainty of the expected CX flux, we cannot rule out the CX scenario with this level of agreement. In addition, there are two pieces of observational evidence supporting the CX scenario. First, optically-emitting clouds, which we think are the major electron donors, are seen only in the BEK, whereas such clouds are not detected in the other regions including the NK and the Einstein FPCS field of view. Second, the N/O and $\mathrm{Ne} / \mathrm{O}$ abundance ratios based on $\mathrm{CX}$ fluxes in the He $\alpha$ triplets for the BEK region are consistent with previous $\mathrm{X}$-ray measurements $(\mathrm{N} / \mathrm{O} \sim 1$ and $\mathrm{Ne} / \mathrm{O} \sim 2$, respectively). Here, we applied the same method used in Liu et al. (2011) who modeled He $\alpha$ triplets with a thermal emission model plus Gaussians to represent the CX emission for the starburst galaxy M82. In this context, we conclude that CX emission is a promising mechanism for explaining the anomalous $\mathrm{F} / \mathrm{R}$ ratios observed in Puppis A.

One might expect to find other spectral signatures of $\mathrm{CX}$ in addition to the $\mathrm{He} \alpha$ line ratios. However, CX spectral properties are strongly dependent on $V_{\mathrm{r}}$ and the target neutrals (e.g., Beiersdorfer, et al. 2001, 2003), and investigation of CX emission is still ongoing. Therefore, further discussion calls for more sophisticated CX emission modeling, which is beyond the scope of this paper and is left as future work.

\section{CONCLUSIONS}

High-resolution X-ray spectra of the cloud-shock interaction regions, the BEK and the NK, in Puppis A have revealed anomalous $\mathrm{He} \alpha$ triplet ratios: in particular, the $\mathrm{O} \operatorname{He} \alpha \mathrm{F} / \mathrm{R}$ line ratios are found to be $\sim 2$ in the BEK. This anomalous ratio can be naturally interpreted as the result of the presence of CX emission, although resonance-scattering effects might be non-negligible.

Future observations with the non-dispersive Soft Xray Spectrometer (SXS: Mitsuda et al. 2010) onboard the Astro- $H$ satellite (Takahashi et al. 2010) will allow for further tests for the CX scenario and other possibilities. While the RGS can perform high-resolution spectroscopy only for bright knotty features, the SXS with expected spectral resolution of $4 \mathrm{eV}$ FWHM and spatial resolution of $1^{\prime}$ HPD (Serlemitsos et al. 2010) will enable us to reveal a spatial distribution of the $\mathrm{F} / \mathrm{R}$ ratios of the knotty features and their surroundings. The CX scenario can then be examined by seeing if there is a spatial correlation between the $\mathrm{F} / \mathrm{R}$ ratios and optical clouds which we here consider to be main electron donors. We expect that other large Galactic SNRs, especially the Cygnus Loop introduced in Section 1, would be also promising sites to check the presence of CX X-ray emission.

We would like to thank Prof. H. Tanuma for fruitful discussions about the laboratory experiments of CX $\mathrm{X}$-ray emission. We also thank the referee for numerous comments which improved the quality of the paper. S.K. and S.Y. are supported by the Special Postdoctoral Researchers Program in RIKEN. This work is partly 
the Ministry of Education, Culture, Sports, Science and Technology (23000004).

\section{REFERENCES}

Arendt, R. G., Dwek, E., Blair, W. P., Ghavamian, P., Hwang, U., Long, K. S., Petre, R., Rho, J., \& Winkler, P. F. 2010, ApJ, 725,585

Beiersdorfer, P., Lisse, C. M., Olson, R. E., Brown, G. V., Chen, H. 2001, ApJ, 549, L147

Beiersdorfer, P., et al. 2003, Science, 300, 1558

Blair, W. P., Raymond, J. C., Long, K. S., \& Kriss, G. A. 1995, ApJ, 454, L35

Bodewits, D., et al. 2007, A\&A, 469, 1183

Borkowski, K. J., Lyerly W. J., \& Reynolds, S. P. 2001, ApJ, 548, 820

Broersen, S., Vink, J., Kaastra, J., \& Raymond, J. 2011, A\&A, 535, 11

den Herder, J. W., et al. 2001, A\&A, 365, L7

Dere, K.P., Landi, E., Young, P.R., Del Zanna, G., Landini, M., \& Mason, H.E. 2009, A\&A, 498, 915

van der Heyden, K. J., Bleeker, J. A. M., Kaastra, J. S., \& Vink, J. 2003, A\&A, 406, 141

Dubner, G. M., \& Arnal, E. M. 1988, A\&AS, 75, 363

Flanagan, K. A., et al. 2004, ApJ, 605, 230

Gillaspy, J. D., Lin, T., Tedesco, L., Tan, J. N., Pomeroy, J. M., Laming, J. M., Brickhouse, N., Chen, G.-X., \& Silver, E. 2011, ApJ, 728, 132

Hwang, U., Flanagan, K. A., \& Petre, R. 2005, ApJ, 635, 355

Hwang, U., Petre, R., \& Flanagan, K. A. 2008, ApJ, 676, 378

Kaastra, J. S., Mewe, R., 1995, A\&A, 302, L13

Kaastra, J. S., Mewe, R., Liedahl, D. A., Singh, K. P., White, N. E., \& Drake, S. A. 1996, A\&A, 314, 547

Katsuda, S., Hwang, U., Petre, R., Park, S., Mori, K., \& Tsunemi, H. 2010, ApJ, 714, 1725

Katsuda, S., et al. 2011, ApJ, 730, 24

Kirshner, R. P., \& Chevalier, R. A. 1978, A\&A, 67, 267

Lallement, R. 2004, A\&A, 422, 391

Liedahl, D., Kahn, S. M., Osterheld, A. L., \& Goldstein, W. H. 1990, ApJ, 350, L37

Liu, J., Mao, S., \& Wang, Q. D. 2011, MNRAS, 415, L64
McClure, G. W. 1966, Physical Review, 148, 47

Mitsuda, K., et al. 2010, SPIE, 7732, 773211

Petre, R., Kriss, G., A., Winkler, P. F., \& Canizares, C. R. 1982, ApJ, 258, 22

Porquet, D., Dubau, J., \& Grosso, N. 2010, Space Sci. Rev., 157, 103

Rasmussen, A. P., Behar, E., Kahn, S. M., den Herder, J. W., \& van der Heyden, K. 2001, A\&A, 365, L231

Reynoso, E. M., Green, A. J., Jhonston, S., Dubner, G. M., Giacani, E. B., \& Goss, W. M. 2003, MNRAS, 345, 671

Read, A., M., \& Ponman, T. J. 2003, A\&A, 409, 395

Serlemitsos, P. J., Boldt, E. A., Holt, S. S., Ramaty, R., \& Brisken, A. F. 1973, ApJ, 184, L1

Serlemitsos, P. J., Soong, Y., Okajima, T., \& Hahne, D.J. 2010, SPIE, 7732, 77320

Smith, R. K., Brickhouse, N. S., Liedahl, D. A., \& Raymond, J. C. 2001, ApJ, 556, L91

Strüder, L., et al. 2001, A\&A, 365, L18

Takahashi, T., et al. 2010, SPIE, 7732, 77320Z

Teske, R. G., \& Petre, R. 1987, ApJ, 314, 673

Turner, M. J. L., et al. 2001, A\&A, 365, L27

Uchida et al. 2012, PASJ submitted.

Vink, J., Laming, J. M., Gu, M. F., Rasmussen, A., \& Kaastra, J. S. 2003, ApJ, 587, L31

Winkler, P. F., Clark, G. W., Markert, T. H., Petre, R., \& Canizares, C. R. 1981a, ApJ, 245, 574

Winkler, P. F., Clark, G. W., Markert, T. H., Kalata, K. Schnopper, H. W., \& Canizares, C. R. 1981b, ApJ, 246, 27 Winkler, P. F., Tuttle, J. H., Kirshner, R. P., \& Irwin, M., J. 1988, in IAU Colloq. 101: Supernova Remnants and the Interstellar Medium, ed. R. S. Roger \& T. L. Landecker, 65

Wise, M. W., \& Sarazin, C. L. 1989, ApJ, 345, 384

Yamaguchi, H., Ozawa, M., Koyama, K., Masai, K., Hiraga, J. S., Ozaki, M., \& Yonetoku, D. 2009, ApJ, 705, L6 